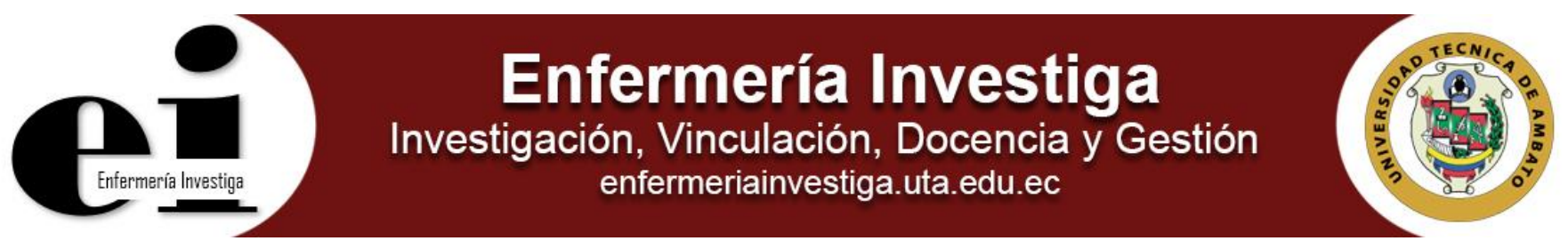

DOI: http://dx.doi.org/10.29033/ei.v3n3.2018.03

Artículo original

\title{
Hallazgos endoscópicos en fallecidos por sangrado digestivo alto no variceal por desequilibrio ácido péptico
}

Endoscopic findings in deceased due to high digestive bleeding no variceal due to peptic acid imbalance

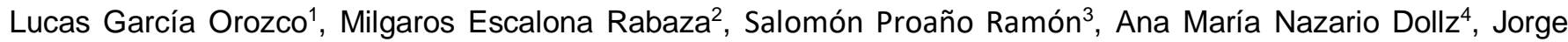
Isaac Sánchez Miño ${ }^{5}$, Enrique Alejandro Rosero Moreno ${ }^{6}$, Fabián Eduardo Yépez Yerovi ${ }^{5}$

${ }^{1}$ Hospital Básico “Asdrubal de la Torre” - Servicio de Cirugía General - Cotacachi - Ibarra - Ecuador.

2 Universidad Técnica de Ambato - Facultad de Ciencias de la Salud - Carrera de Enfermería - Ambato - Tungurahua - Ecuador.

${ }^{3}$ Gerencia Dirección Distrital 10D03 - Cotacachi - Ibarra - Ecuador.

${ }^{4}$ Universidad de Ciencias Médicas de Santiago de Cuba -Carrera de Medicina - Santiago de Cuba - Cuba.

5 Universidad Técnica de Ambato - Facultad de Ciencias de la Salud - Carrera de Medicina - Ambato - Tungurahua - Ecuador.

6 Hospital Básico "Asdrubal de la Torre" - Cotacachi - Ibarra - Ecuador.

García L, Escalona M, Proaño S, Nazario Ana, Sánchez J, Rosero E, Yépez F. Hallazgos endoscópicos en fallecidos por sangrado digestivo alto no variceal por desequilibrio ácido péptico. Enferm Inv. 2018;3(3):122-128.

2477-9172 / 2550-6692 Derechos Reservados @ 2018 Universidad Técnica de Ambato, Carrera de Enfermería. Este es un artículo de acceso abierto distribuido bajo los términos de la Licencia Creative Commons, que permite uso ilimitado, distribución y reproducción en cualquier medio, siempre que la obra original es debidamente citada.

\section{Historia:}

Recibido: 8 diciembre 2017

Revisado: 28 febrero 2018

Aceptado: 22 agosto 2018

Palabras Claves: Hemorragia digestiva alta; enfermedad acido péptica; resangrado; terapia intervencionista

Keywords: Upper gastrointestinal bleeding; peptic acid disease; bleeding; interventional therapy

\begin{abstract}
Resumen
Introducción: La hemorragia digestiva alta es asumida como una emergencia patológica de causas diversas, que deviene en complicaciones fatales con relativa frecuencia.

Objetivo: Identificar los hallazgos endoscópicos presentes en pacientes con desenlaces fatales (muerte) por HDA de etiología ácido-páptica, durante 3 años consecutivos.

Métodos: Se realizó una investigación descriptiva de serie de casos para valorar los hallazgos endoscópicos en fallecidos por sangrado digestivo alto no variceal por desequilibrio ácido péptico en 25 pacientes egresados de los Servicios de Cuidados Intensivos e Intermedios y de Cirugía General del Hospital Provincial Clínico Quirúrgico Docente "Saturnino Lora Torres" de Santiago de Cuba, durante el trienio 2012 (enero) - 2015 (diciembre).

Resultados: Los ancianos mayores de 66 años resultaron el grupo de edad preponderante y del sexo masculino. Los antecedentes predominantes en la serie estuvieron liderados por el consumo de AINE, antecedente de enfermedad acido-péptica conocida e hipertensión arterial. El diagnóstico más utilizado resultó el clínico endoscópico (56\%). Conclusiones: Los procesos ulcerosos duodenales con tamaños inferiores a $2 \mathrm{~cm}$ resultaron los más frecuentes y con predominio de la clasificación de Forrest II-b, lo cual expresa por si solo su importancia en cuanto al riesgo de resangrado y de fatales desenlaces, pudiendo servir los datos para futuros estudios similares al relacionar los mismos con resultados de terapias intervencionistas.
\end{abstract}

\begin{abstract}
Introduction: Upper gastrointestinal hemorrhage is assumed as a pathological emergency of diverse causes, which results in fatal complications with relative frequency.

Objective: To identify the endoscopic findings present in patients with fatal outcomes (death) due to HDA of acid-páptica etiology, during 3 consecutive years.

Methods: A descriptive investigation of case series was carried out to evaluate the endoscopic findings in deceased persons due to high non-variceal digestive bleeding due to peptic acid imbalance in 25 patients graduated from the Intensive and Intermediate Care Services and General Surgery of the Hospital Provincial Clínico Quirúrgico Docente "Saturnino Lora Torres" of Santiago de Cuba, during the triennium 2012 (January) - 2015 (December).

Results: The elderly over 66 years were the predominant age group and male. The predominant antecedents in the series were led by the consumption of NSAIDs, a history of known acidpeptic disease and arterial hypertension. The most used diagnosis was the endoscopic clinician (56\%). Conclusions: The duodenal ulcer processes with sizes smaller than $2 \mathrm{~cm}$ were the most frequent and with a predominance of the Forrest II-b classification, which in itself expresses its importance in terms of risk of rebleeding and fatal outcomes, which can serve for future similar studies when relating them to the results of interventional therapies.
\end{abstract}

Autor de correspondencia:

Lucas García Orozco. Servicio de Cirugía General, Hospital Básico Asdrúbal de la Torre, Avenida Paco Moncayo s/n. Cotacachi, Teléfono: +593 06 2554118, Ibarra, Ecuador. E-mail: gamilulu7952@gmail.com 


\section{Introducción}

La hemorragia digestiva alta (HDA), frecuentemente abordada como un síndrome y referida a toda pérdida hemática de volumen suficiente como para producir hematemesis, melena o ambas, con origen entre el esfínter esofágico superior y el ángulo de Treitz; se enfoca por lo general en dos grandes grupos de causas: De etiología variceal y no variceal, siendo estas últimas enfocadas como una emergencia médico-quirúrgica y responsable de la mayoría de complicaciones y desenlaces fatales. Los niveles de afección y las variantes patológicas descritas han sido diversos, pero merecen distinción las causas de origen ácido péptico, localizadas en estómago y duodeno y por fortuna se dispone en la actualidad de medios diagnósticos que nos pueden orientar hacia un curso desagradablemente fatal luego de que esta se instaura. ${ }^{1,2}$

El comportamiento mundial de esta entidad continúa asombrando, condicionado por varios aspectos resaltando varios como situación geográfica, hábitos sanitarios y patrones culturales.

Variable también han sido sus formas de instauración, siendo las agudas las más frecuentes que generan repercusiones sistémicas en ocasiones difíciles de reajustar, incluso los avances científicos resultan limitados con frecuencia, asumiendo enfoques intervencionistas, pero pueden informar cambios que sugieren comportamientos desagradables, sobre todo en poblaciones con determinados factores de riesgo, como son los seniles y los pacientes con enfermedades asociadas. ${ }^{3}$

El sitio de origen de la hemorragia puede variar pudiendo ser a nivel gastroduodenal, siendo las úlceras péptica gástrica y péptica duodenal sus principales exponentes y esta última es preponderante en el sitio donde se desarrolla el trabajo (Cuba), mostrando similares comportamientos en otras zonas del orbe; seguidas por las várices esofagogástricas erosionadas, presentes en enfermos con hipertensión portal, el síndrome de MalloryWeiss y la gastritis hemorrágica con sus variantes etiopatológicas, desde las producidas por hábitos inadecuados de alimentación hasta las que se presentan en el curso de hospitalizaciones por infecciones graves, quemaduras, insuficiencia respiratoria con ventilación artificial y períodos posoperatorios complicados. ${ }^{3,4}$

Esquemas de organización diferentes entre sí han sido propuestos para perfeccionar su manejo, no obstante nada supera la evaluación rápida, pero sistemática del problema y un tratamiento eficaz y oportuno, preferentemente en forma interdisciplinaria (cirujano, gastroenterólogo, endoscopista, imagenólogo intervencionista y patólogo clínico), con imprescindible apoyo de la unidad de hemoterapias. ${ }^{5-8}$

El tratamiento deviene a ser complemento que generará mejoría en estos enfermos, pero la decisión del momento y el procedimiento intervencionista a emplear debe ser altamente individualizada según el estado del enfermo, edad, el tipo de lesión causante de la hemorragia, entre otros. La determinación de operar corresponde al cirujano, pero la opinión del internista gastroenterólogo merece ser escuchada.

Sin duda alguna, el tratamiento adecuado de personas con HDA no varicosa es un reto para cualquier servicio quirúrgico; y en este caso, el empleo de la terapéutica endoscópica constituye la esencia de la problemática a desarrollar, con sus posibles efectos en cuanto a estándares de seguridad, vista desde indicadores de hallazgos. En este sentido existen diversos comportamientos que han de sustentarse en hallazgos preoperatorios endoscópicos y que en este trabajo han de exponerse basado en los detectados en enfermos fallecidos. ${ }^{9,10}$

Todo lo anterior justificó la necesidad de profundizar en los principales aspectos cognoscitivos relacionados con los hallazgos endoscópicos en el curso de HDA no varicosa por desequilibrios acido-pépticos que fueron evidenciados previamente en enfermos fallecidos por esta razón, pertenecientes al Hospital Provincial Clínico Quirúrgico Docente "Saturnino Lora Torres" de la provincia de Santiago de Cuba, Cuba, durante en el trienio 2012 (enero)- 2015 (diciembre).

\section{Materiales y métodos}

Se realizó una investigación aplicada y descriptiva de serie de casos para identificar los hallazgos endoscópicos en pacientes posteriormente fallecidos por HDA durante enero de 2012 hasta diciembre de 2015, ambos meses inclusive; previa comunicación al Consejo Científico del centro hospitalario para cumplir adecuadamente con la aceptación del mismo al considerar los principios bioéticos básicos y el respeto a las normas establecidas.

Como universo de estudio resultaron todos los pacientes fallecidos por HDA no varicosa debido a enfermedad ácido péptica, admitidos en la citada institución durante el trienio señalado, resultando finalmente en 25 , según los siguientes criterios de inclusión:

1- Fallecidos por HDA no varicosa en el Hospital Provincial Clínico-Quirúrgico Docente "Saturnino Lora Torres" desde enero de 2012 a diciembre de 2015, en las unidades de Cuidados Intensivos e Intermedios y el Servicio de Cirugía General.

2- Diagnóstico confirmado de enfermedad ácido péptica al egreso. 
Para obtener los datos necesarios se revisaron datos estadísticos internacionales, del hemisferio, el país y la provincial de Santiago de Cuba. Además se estudiaron las historias clínicas hospitalarias, informes endoscópicos y anatomopatológicos, así como certificados de defunción. También se realizó una amplia revisión bibliográfica sobre el tema evaluando lo publicado en bases de datos validadas por expertos: LILACS, SciElo, PubMed, Scopus y otras, para revisar importantes artículos relacionados con la temática.

La información finalmente se procesó en una computadora HP Corel7, habilitada con sistema operativo Microsoft Office Word 2010 y el software estadístico profesional SPSS, versión 21 portable y se utilizaron medidas de resumen para variables cualitativas.

El análisis y la discusión de los resultados se efectuaron a través del método deductivo e inductivo, previamente mostrados en tablas, así como comparados con los descritos por otros autores lo que finalmente permitió formular conclusiones y enunciar respectivas recomendaciones.

\section{Resultados}

La HDA conlleva en ocasiones a desenlaces fatales, incluso difíciles de modificar en su curso a finales no deseados, aunque en la actualidad se le brinda capital importancia a los hallazgos endoscópicos una vez que se instaure esta. Tal es el actual desarrollo que la endoscopia intervencionista pasa a ocupar posiciones cimeras en las diferentes posibilidades terapéuticas a emplear para contrarrestarla. ${ }^{11,12}$

En nuestra serie existió predominio del sexo masculino y con una razón cercana al doble (60\% vs $40 \%)$, prevaleciendo en ambos los pacientes adultos mayores con edades superiores representados por el $60 \%$ de los casos.

Tabla 1: Pacientes según sexo y grupos de edades. Hospital Provincial Clínico-Quirúrgico Docente "Saturnino Lora Torres". Enero de 2012 - diciembre de 2015.

\begin{tabular}{|c|c|c|c|c|c|c|}
\hline \multirow{3}{*}{$\begin{array}{l}\text { Grupos de edades } \\
\text { (en años) }\end{array}$} & \multicolumn{4}{|c|}{ Sexo } & \multicolumn{2}{|c|}{ Total } \\
\hline & \multicolumn{2}{|c|}{ Masculino } & \multicolumn{2}{|c|}{ Femenino } & \multirow[b]{2}{*}{ n. } & \multirow[b]{2}{*}{$\%$} \\
\hline & n. & $\%$ & n. & $\%$ & & \\
\hline $16-25$ & - & - & - & - & - & - \\
\hline $26-35$ & - & - & - & - & - & - \\
\hline $36-45$ & - & - & 1 & 4 & 1 & 4 \\
\hline 46-55 & 2 & 8 & 1 & 4 & 3 & 12 \\
\hline 56-65 & 2 & 8 & 4 & 16 & 6 & 24 \\
\hline 66 y más & 6 & 24 & 9 & 36 & 15 & 60 \\
\hline Total & 10 & 40 & 15 & 60 & 25 & 100 \\
\hline
\end{tabular}

Se consideran numerosos antecedentes que guardan estrecha relación con la posibilidad de que se presente una $H A D$, pero en nuestra casuística los referidos en la tabla 2, resultaron los preponderantes, sobresaliendo por sus frecuencias el consumo de AINES, el antecedente de Hipertensión Arterial (HTA) y el consumo crónico de tabaco o sus similares $(21.8 \%, 16.8 \%, 12.9 \%)$ respectivamente. 
Tabla 2: Antecedentes personales de interés.

\begin{tabular}{|c|c|c|c|}
\hline \multicolumn{2}{|c|}{ Antecedentes personales de interés } & n. & $\%$ \\
\hline \multicolumn{2}{|c|}{ Úlcera gástrica } & 1 & 1 \\
\hline \multicolumn{2}{|c|}{ Úlcera duodenal } & 7 & 6.9 \\
\hline \multicolumn{2}{|c|}{ Gastritis } & 10 & 9.9 \\
\hline \multicolumn{2}{|c|}{ HDA previa de causa desconocida } & 4 & 4 \\
\hline \multirow{7}{*}{ Otros } & AINES & 22 & 21.8 \\
\hline & Hábito enólico & 3 & 3 \\
\hline & Tabaquismo crónico & 13 & 12.9 \\
\hline & Anticoagulantes & 3 & 3 \\
\hline & Hipertensión arterial & 17 & 16.8 \\
\hline & Cardiopatía & 8 & 7.9 \\
\hline & $\begin{array}{l}\text { Insuficiencia renal } \\
\text { crónica }\end{array}$ & 4 & 4 \\
\hline & Total & 101 & 100 \\
\hline
\end{tabular}

A la luz de los avances es aceptada la endoscopia digestiva superior como el "gold standard" en el diagnóstico de HDA, siempre que no se constaten contraindicaciones, y en la presente serie el comportamiento no resultó diferente, siendo mayoritario el diagnóstico clínico endoscópico (56\%); aunque existen otros métodos que pudieran utilizarse como la arteriografía, enteroscopia de doble balón, gammagrafía, cápsula endoscópica, entre otros; y menos utilizados como el diagnóstico clínico imagenológico, o simplemente el clínico los que resultaron menos en la serie..$^{5,10-16}$

Figura 1. Métodos utilizados para realizar el diagnóstico.

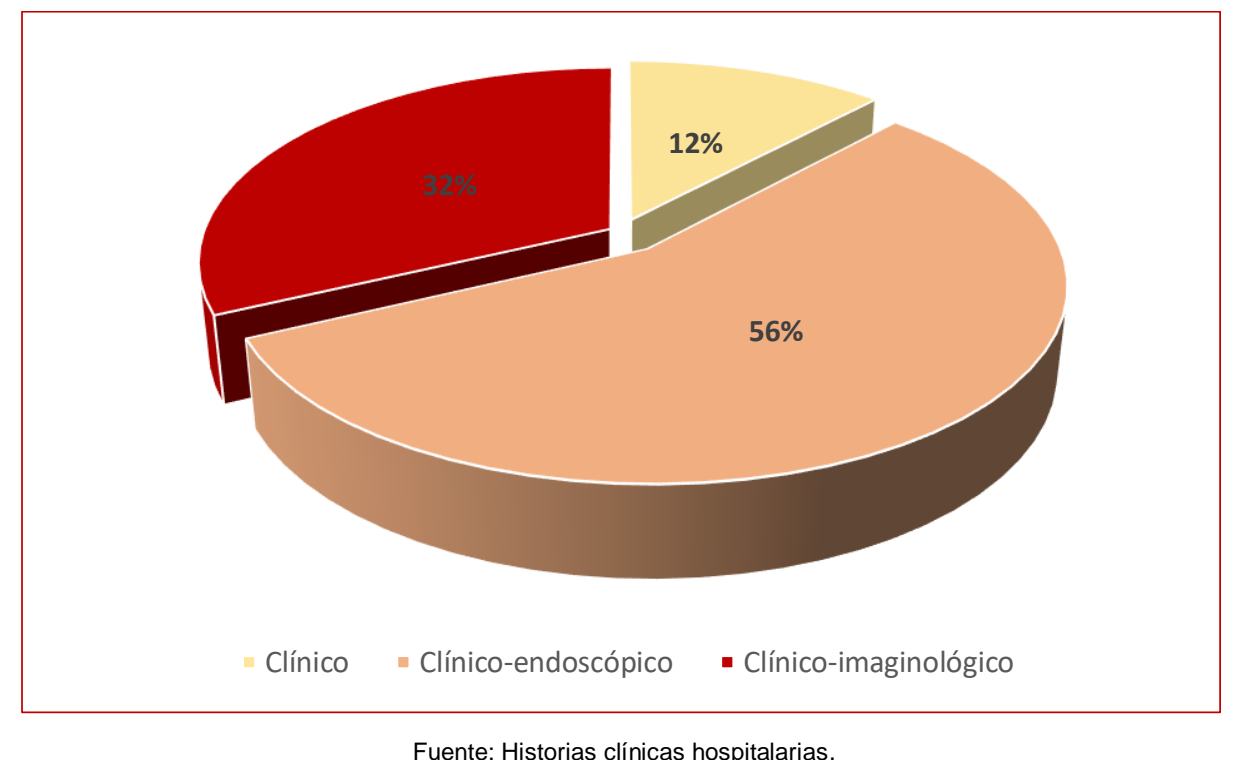

La actual investigación permitió determinar además que la localización predominante de los procesos acido pépticos evidenciados estuvieron representados mayoritariamente por erosiones circunscritas en la vertiente duodenal y con tamaños inferiores a $2 \mathrm{~cm}$. También estos tamaños de úlceras localizadas en el estómago fueron los predominantes. 
Tabla 3: Localización y extensión del proceso actual.

\begin{tabular}{|c|c|c|c|}
\hline \multicolumn{2}{|c|}{ Localización del proceso actual } & n. & $\%$ \\
\hline \multicolumn{2}{|c|}{ Gástrico difuso } & 7 & 28 \\
\hline \multirow{2}{*}{ Gástrico preciso } & $\leq 2 \mathrm{~cm}$ & 4 & 16 \\
\hline & $>2 \mathrm{~cm}$ & 2 & 8 \\
\hline \multicolumn{2}{|c|}{ Duodenal difuso } & - & - \\
\hline \multirow{2}{*}{ Duodenal preciso } & $\leq 2 \mathrm{~cm}$ & 9 & 36 \\
\hline & $>2 \mathrm{~cm}$ & 1 & 4 \\
\hline \multicolumn{2}{|c|}{ Combinación } & 2 & 8 \\
\hline & Total & 25 & 100 \\
\hline
\end{tabular}

El tamaño de la úlcera ha devenido en los últimos años en un factor a considerar al identificar un proceso erosivo circunscrito al predecir la intensidad del sangrado y la posibilidad de resangrado y por consiguiente de muerte, aún consecutivo a la aplicación de un tratamiento hemostático endoscópico.

Algunas series le confieren importancia a las úlceras mayores de $2 \mathrm{~cm}$ de diámetro pues pueden potencialmente producir erosión vascular en contigüidad y afectar vasos de importante calibre, principalmente aquellas localizadas en la cara posterior del bulbo duodenal por su cercanía a la arteria gastroduodenal y en la porción proximal de la curvatura menor gástrica por su proximidad a la arteria gástrica izquierda. ${ }^{10-19}$

Del total de pacientes fallecidos solo en 21 se pudo aplicar la clasificación de Forrest, mostrando los siguientes resultados:

Tabla 4: Clasificación de Forrest.

\begin{tabular}{lccc}
\hline \multicolumn{2}{l}{ Clasificación de Forrest } & n. & $\%$ \\
\hline Forrest- I & a & 1 & 4.8 \\
Forrest- II & b & 2 & 9.5 \\
& a & 3 & 14.3 \\
Forrest- III & b & 10 & 47.6 \\
Total & c & 4 & 19 \\
\hline \multicolumn{2}{c}{ Fuente: Historias clínicas hospitalarias. }
\end{tabular}

La anterior distribución se utiliza para evidenciar el estado gastroduodenal secundario a la presencia de úlceras indistintamente en una región u otra y además predice el riesgo de resangrado y por tanto es un indicador relativo de resangrado e indirectamente de mortalidad.

En el presente trabajo se evidenció que existió predominio del grupo II-b representado por $47.6 \%$ y ello llama la atención pues esta serie en su totalidad versa en torno a pacientes que posteriormente resultaron fallecidos, es decir la categoría antes citada expresa riesgo de resangrado, relacionado además con complicaciones fatales.

\section{Discusión}

El comportamiento del sexo en el presente estudio concuerda con lo declarado en algunas series incluso en edades similares. Pudiera explicarse tal comportamiento atendiendo a varios factores: La población actual en Cuba se considera envejecida y al mejorarse la esperanza de vida tal comportamiento tiende hacerse más significativo. Por tal motivo se comprende que en uno de cada dos gerontes que acuden a la consulta de geriatría por cualquier motivo, se diagnostica en uno de estos una enfermedad ácido péptica, siendo la úlcera péptica la más frecuente..$^{9,11}$ Otro dato significativo pudiera explicar que con la edad algunas actividades enzimáticas se alteran a consecuencia de errores existentes entre el ADN, el ARN y la síntesis proteica, lo que puede ocasionar desequilibrios ácido-básicos y ello intentaría explicar porque estas personas son más vulnerables a padecer trastornos estomacales diversos y por consiguiente presentar HDA con relativa frecuencia. ${ }^{12,14}$

Pudiera intentarse añadir a las evidencias reflejadas en la tabla de los antecedentes, el dato de que aproximadamente el $25 \%$ de los consumidores crónicos de AINES sufren reacciones adversas al medicamento, 
entre $10-30 \%$ presentan una úlcera péptica a los seis meses de tratamiento continuo con antiinflamatorios no esteroideos y generalmente en ellos se cuadruplica el riesgo de sangrado digestivo en contraste con la población en general. Por otro lado en la población estudiada la HTA representa la enfermedad crónica no trasmisible más frecuente y el tabaquismo representa uno de los tres primeros hábitos tóxicos. ${ }^{3}$

Con relación al diagnóstico, se debe acotar que las ventajas del método clínico endoscópico son indiscutibles, pues además de especificar en tiempo real el estado del nivel digestivo hacia el cual esté orientada, es un método que posibilita realizar terapéutica e incluso complementar una intervención quirúrgica.

Los hallazgos sobre la localización del proceso sangrante concuerdan con otros reportes al enunciar a la úlcera péptica como la causa más frecuente de HDA en aproximadamente más de la mitad de los casos estudiados y de estas a la duodenal con frecuencia mayor que la gástrica. Al relacionar estas evidencias con las edades de los pacientes estudiados, varios sustentos podemos citar: En la medida que la edad se incrementa, el consumo de AINE se intensifica y tiende hacerse prolongado con los subsiguientes efectos colaterales, por otro lado con la edad se eleva la periodicidad de reflujo duodenobiliar, cuya acción detergente sobre el moco gástrico disminuye su eficacia como barrera protectora; en los ancianos las alteraciones vasculares y la aminoración de la velocidad de recambio de las células epiteliales se hace notoria lo cual contribuye a la aparición de procesos acido-pépticos. ${ }^{17-20}$

Existen otros índices de confiabilidad diferente tales como el Índice de Rockall, Clasificación de Sakita, Escala de Blatchford, entre otros, que pretenden establecer escalas pronósticas y por consiguiente adecuar el tipo de tratamiento a seguir en cada caso particular. , $^{5,20-22}$

La importancia de la clasificación seleccionada para evaluar las evidencias y realizar nuestro análisis es que la clasificación de Forrest se fundamenta exclusivamente en los hallazgos endoscópicos y solo permite pronosticar el riesgo de resangrado, lo cual es de vital importancia si consideramos el peligro que encierra la HDA.

\section{Conclusiones}

Con la presente investigación pudimos arribar a las siguientes conclusiones:

1. Predominaron los hombres, en una proporción cercana al doble respecto a las mujeres, siendo mayoritarios en ambos sexos los pacientes seniles.

2. Los principales factores de riesgos detectados mostraban relación con antecedentes patológicos familiares de importancia y hábitos inadecuados de vida, siendo el más común el consumo de antiinflamatorios no esteroideos.

3. Los procesos acido-pépticos más frecuentes resultaron ser en orden de frecuencia las úlceras duodenales, las gastritis y las úlceras gástricas, siendo de tamaños inferiores a $2 \mathrm{~cm}$ los de tipo erosivo circunscrito.

4. Resultó mayoritario el diagnóstico clínico complementado con la exploración endoscópica, seguido del diagnóstico clínico apoyado en el examen imagenológico y finalmente solo mediante el diagnóstico clínico.

5. El principal hallazgo evidenciado por endoscopias mostró como predominantes a los procesos mórbidos de tipo ácido-pépticos gástricos y duodenales, y permitió según la clasificación de Forrest determinar que el subtipo (III-b) predominó.

\section{Conflicto de intereses}

Ninguno declarado por los autores.

\section{Financiación}

Autofinanciado.

\section{Agradecimientos}

Ninguno declarado por los autores

\section{Referencias}

1. Ahmed A, Armstrong M, Robertson I, Morris AJ, Blatchford O, Stanley AJ. Upper gastrointestinal bleeding in Scotland 2000-2010: Improved outcomes but a significant weekend effect. World J Gastroenterol. 2015;21(38):10890-7.

2. Rodríguez Fernández Z, Acosta González D, Fong Estrada J. Conducta ante la hemorragia digestiva alta por úlcera gastroduodenal en el anciano: consideraciones actuales. Rev Cubana Cir [serie en Internet] 2008 [citado 2 Abr 2010];49. Disponible en: http://scielo.sld.cu/scielo.php?script=sci_arttext\&pid=S0034-74932010000100011\&lng=es.

3. Gaudong Mbethe GL, Mounguengui D, Ondounda M, Magne C, Bignoumbra R, Ntsoumou S, et al. [Epidemiology of upper gastrointestinal bleeding in Gabon]. Med Sante Trop. 2014;24 (4):441-3.

4. Moayyedi P, Soo S, Deeks J. Eradication of Helicobacter pylori for non-ulcer dyspepsia. Cochrane Database Syst Rev 2006;2:CD002096 [citado 15 Dic 2011]. Disponible en: http://www.ncbi.nlm.nih.gov/pubmed/15674892.

5. Tiuca N, Sztogrin W. The news of treatment of variceal upper gastrointestinal bleeding. J Med Life. 2011;4(4):395-8.

6. Lee SY. There Is No Reason to Delay Helicobacter pylori Eradication after Treatment for Upper Gastrointestinal Bleeding. Gut Liver. 2015;9(3):259-60.

7. Bai Y, Li ZS. Guidelines for the diagnosis and treatment of acute non-variceal upper gastrointestinal bleeding (2015, Nanchang, China). J Dig Dis. 2016;17(2):79-87.

8. Jenkins SA, Shields R. Drug treatment for acute upper gastrointestinal bleeding. BMJ. 1992;304(6829):778.

9. Miyamoto M, Haruma K, Okamoto T, Higashi Y, Hidaka T, Manabe N. Continuous proton pump inhibitor treatment decreases upper gastrointestinal bleeding and related death in rural area in Japan. J Gastroenterol Hepatol. 2012;27(2):372-7. 
10. Kurt M, Disibeyaz S, Akdogan M, Sasmaz N, Aksu S, Haznedaroglu IC. Endoscopic application of ankaferd blood stopper as a novel experimental treatment modality for upper gastrointestinal bleeding: a case report. Am J Gastroenterol. 2008;103(8):2156-8.

11. Botianu A, Matei D, Tantau M, Acalovschi M. Mortality and need of surgical treatment in acute upper gastrointestinal bleeding: a one year study in a tertiary center with a 24 hours / day-7 days / week endoscopy call. Has anything changed? Chirurgia (Bucur). 2013;108(3):312-8.

12. Cioni G, Cristani A, Vignoli A, Ventura E. High D-dimer levels: a possible index of risk of overt disseminated intravascular coagulation and/or digestive bleeding in advanced liver cirrhosis? Recenti Prog Med. 1994;85(4):230-4.

13. Greve E, Moussata D, Gaudin JL, Lapalus MG, Giraud S, Dupuis-Girod S, et al. High diagnostic and clinical impact of small-bowel capsule endoscopy in patients with hereditary hemorrhagic telangiectasia with overt digestive bleeding and/or severe anemia. Gastrointest Endosc. 2010;71(4):760-7.

14. Fleitas Pérez O, Muarra HD, Gil Casas B, Roye Reina Y. Morbimortalidad por sangramiento digestivo alto en el adulto mayor. Análisis de 3 años.

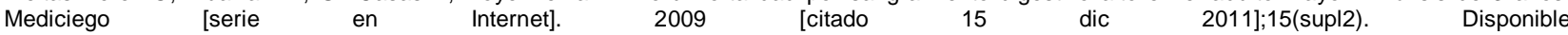
en:http://bvs.sld.cu/revistas/mciego/vol15 supl2 09/articulos/a5 v15 supl209. htm.

15. Ramírez GA, Loyarte LY. Resultados del tratamiento quirúrgico de la úlcera péptica gastroduodenal en ancianos en el último decenio. Sancti Spíritus: XIX Fórum Nacional de Ciencias Médicas; 2008.

16. Lepore GV, Sampor CV, Storni MP, Calderón Gospodinoff SR. Lesiones endoscópicas más frecuentes en un grupo de pacientes con clínica de hemorragia digestiva alta. Rev Posgrado Vla Cátedra Med 2006;158:5-8.

17. Almeida R, Pérez F, Díaz JO, Martínez JA. Comportamiento de la hemorragia digestiva alta en el Hospital universitario "Calixto García". Revista Cubana de Cirugía 2011;50(1):40-13.

18. Barkun A, Sabbah S, Enns R. The Canadian Registry on Nonvariceal Upper Gastrointestinal Bleeding and Endoscopy (RUGBE): endoscopic hemostasis and proton pump inhibition are associated with improved outcomes in a real-life setting. Am J Gastroenterol 2004;99:1238-46.

19. Machuca MT, Fernández DL, Gómez MN. La endoscopia en la hemorragia digestiva. Rev Posgr Vla Cátedra Med 2008;(180).

20. Abraham Arap JF, Mederos Curbelo ON, García Sierra JC, Gigato Díaz A. Sangramiento digestivo alto. La Habana: Editorial de Ciencias Médicas; 2007. p. 16

21. Xie B, Xiao SC, Zhu SH, Wang GY, Lv KY, Li HY, et al. Risk factors and prognosis of lower digestive tract haemorrhage in severe burns: a 12-year retrospective analysis. Burns. 2011;37(2):328-32.

22. Rosenstock S, Jorgensen T, Bonnevie O, Andersen L. Risk factors for peptic ulcer disease: a population based prospective cohort study comprising 2416 Danish adults. Gut 2003;52:186-93.

Enferm Inv. 3(3):122-128 